\title{
Malignant Priapism Due to Renal Cancer: A Case Report of First Manifestation of Systemic Spread
}

\author{
Jin Zhu ${ }^{1}$; Boxin Xue ${ }^{1}$; Dongrong Yang ${ }^{1}$; Yachen Zang ${ }^{1}$; Yuxi Shan ${ }^{1, *}$ \\ ${ }^{1}$ Department of Urology, the Second Affiliated Hospital of Soochow University, Suzhou City, Jiangsu, China \\ ${ }^{*}$ Corresponding Author: Yuxi Shan, Department of Urology, the Second Affiliated Hospital of Soochow University, Suzhou City, Jiangsu, China. Tel: +86-51267784136, Fax: +86- \\ 51267784136, E-mail: shanyx1002@yahoo.com
}

Received: December 6, 2012; Revised: July 29, 2013; Accepted: November 1, 2014

\begin{abstract}
Introduction: Malignant priapism due to cancers is a rare condition that only 30 cases of kidney cancers were reported. Case Presentation: We report on the first case of simultaneous penile, renal vein, vena cava, liver and ipslateral adrenal gland metastases from primary renal cancer in a 56-year-old man. The only complain of the patient was a history of painful priapism for one month. Corporoglanular shunt surgery was useless. Postoperative imaging showed extensive regional and distal metastases and tumor embolus in vena cava as well as renal and hepatic vein.

Conclusions: Review of literature showed priapism being the first presentation in $20 \%$ to $50 \%$ of cases, almost all cases have shown very poor prognosis. This case report underscored the importance of investigation of primary tumors for patients with malignant priapism.
\end{abstract}

Keywords: Priapism; Metastasis; Renal Cancer

\section{Introduction}

Priapism due to malignancy metastasis to the penis is rare. For the first time in 1938, Peacock named this disease as "malignant priapism" (1). Sporadic cases were also reported. We report on a case of malignant priapism due to renal cancer of which priapism is the only manifestation of the tumor.

\section{Case Presentation}

The patient was a 56-year-old man from rural area of Suzhou city, China. He was referred to our department in August 2011 with increasingly painful erection of the penis for 34 days. The erection was not associated with sexual stimulation. There was no history of hematonosis, tumor, trauma, hypertension or neuropathy, nor did the patient take any medicine in the past three months. On physical examination, the penis erected with an erectile angle of about $90^{\circ}$. The penis shaft was rigid and dropsical. The glans was flaccid with dull-red appearance. Palpation along the length of the penis elicited pain. Chest X-ray was negative. Regular blood test as well as liver and kidney function tests showed normal results. Color Doppler ultrasound of the penis did not show any definite changes. Cavernous blood gas test showed hypoxia, hypercapnia and acidosis. He underwent corporoglanular shunt surgeries four and 14 days after the admission. During the study we found that cavernous body were fibrosised and filled with blood clots. The procedures turned out to be of no improve. Post-operative computed tomography (CT) scan disclosed right renal cancer with embolus involved the right renal vein, hepatic vein and vena cava from the level of common iliac vein to right atrium. Metastatic to liver, right adrenal gland and retroperitoneal lymph nodes were also revealed (Figure 1). Angiographs showed the abnormality of the penile circulation (Figure 2). Knowing his condition, the patient selected to leave hospital and died five weeks later.

\section{Discussion}

Priapism is the situation that the penis keeps erecting for more than four hours without sexual stimulation, which is not a common condition. Eland et al. (2) reported 1.5 cases with priapism in every 100000 people per year. The etiopathogenisis of priapism is still not well known. Common causes are disorders such as sickle cell disease, leukemia, polycythemia, sickle cell anemia, penile metastasis of tumors, pelvic thrombophlebitis, and neurological diseases $(3,4)$. Among these causes, malignant priapism is really rare disease, which is described as prolonged erection due to an invasion of the corpora cavernosa by a malignant neoplasm (5). Yu-Hsiang Lin et al. (6) reviewed the literature and reported a total of 394 cases of secondary penile malignancies up to January 2011 and also reported malignant priapism in $20 \%$ to $50 \%$ of the documented cases. Malignant priapism is considered to be the first manifestation of tumors in $20-53 \%$ of cases (7). 

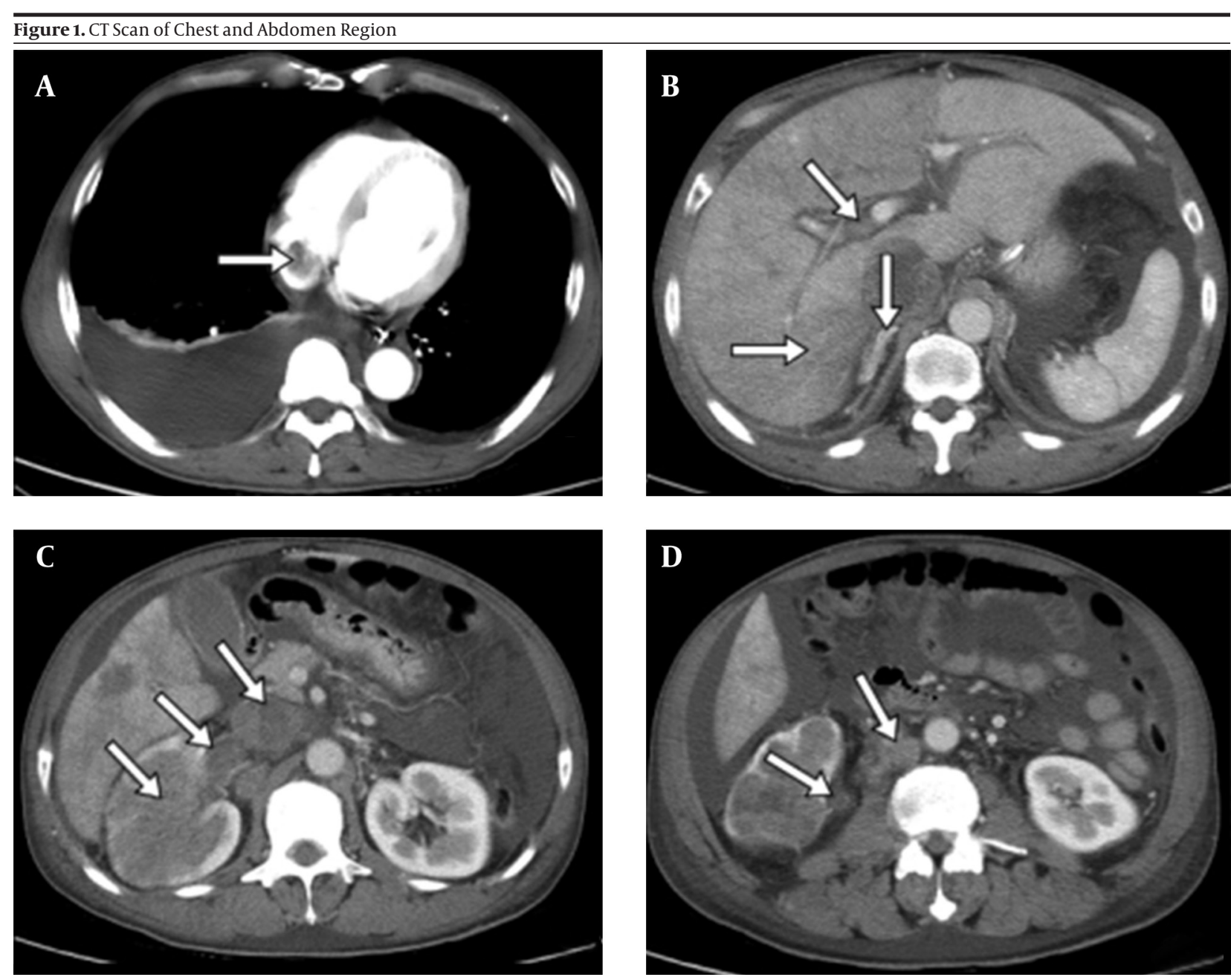

Right renal cancer (C) and metastasis in right atrium (A), liver, hepatic vein, right adrenal (B), right renal vein, vena cava (C), and retroperitoneal lymphnodes (D). The arrows show the primary and metastatic lesions.

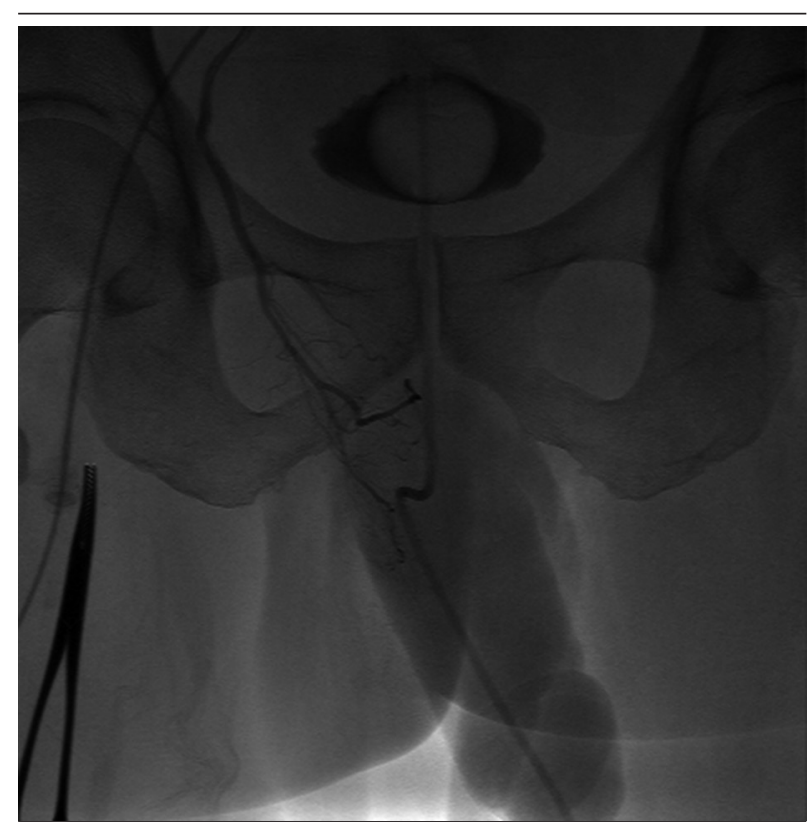

Figure 2. Abnormality of the Penile Circulation
The most frequent primary tumors come from the genitourinary (70\%) and gastrointestinal tracts (23\%). Among these, the bladder cancer (27\%) and prostate cancer (26\%) are the most common primary tumors, followed by the renal cell carcinoma (8-11\%) and testicular germ cell neoplasm (3-10\%), respectively $(5,8)$. How penile metastasis develops is still not quite clear. Direct extension, retrograde venous or lymphatic transport, arterial embolism, implantation and instrumentation are the five most possible mechanisms of tumor spread to the penis (9). It is vital for urologists to be alerted of priapism refractory in regular therapy such as pharmacologic therapies or shunts. Correct diagnosis of such an uncommon condition requires careful examinations. Penile ultrasonography is a useful method to detect the metastatic lesions and is helpful to differentiate high and low flow priapism. Cavernosa blood gases test can distinct between the two types of priapism. We recommend that chest-abdomen-pelvic CT scan be perform immediately because it is reliable for confirming the diagnosis and assessing of disease extension. MRI scan is also a useful alternative (10), but the best diagnosis is obtained through histopathological biopsy. 
Regretfully, in this case we did not take biopsy samples from the cavernosa tissue. Penile metastasis usually represents as the systemically disseminated disease with very poor prognosis. The majority of patients die within 18 months, with the average survival being of nine months. Various treatment modalities for penile metastasis are described as no treatment, local excision, partial or total penectomy, radiotherapy, and chemotherapy, but all these therapies are palliative. Consequently the management should be individualized. If the patients have a short life expectancy or they are asymptomatic, it is reasonable to choose conservative treatment or no treatment. For patients with severe pain or urinary tract symptoms such as retention, total penectomy is the best therapeutic choice. If the tumor diffuses severely, radiotherapy, chemotherapy or supportive treatments to lessen the symptoms should be considered.

\section{Acknowledgements}

We thank Dr. Wei Tao for his help in the writing manuscript.

\section{Authors' Contributions}

Sugery: Jin Zhu, Boxin Xue, Dongrong Yang, Yachen
Zang, Yuxi Shan. Drafting of the manuscript: Jin Zhu. Critical revision of the manuscript for important intellectual content: Boxin Xue, Yuxi Shan.

\section{References}

1. Peacock A. Malignant priapism due to secondary carcinoma in the corpora cavernosum. Northwest Med.1938;37:143-5.

2. Eland IA, van der Lei J, Stricker BH, Sturkenboom MJ. Incidence of priapism in the general population. Urology. 2001;57(5):9702.

3. Campbell MF, Wein AJ, Kavoussi LR. Campbell-Walsh Urology:: W.B. Saunders; 2007.

4. Jiang H, Zhu XW, Shi SF, Xie LP, Teng XD, Chen ZD, et al. Malignant priapism secondary to testicular tumor. Chin Med J (Engl). 2009;122(15):1839-40.

5. Dubocq FM, Tefilli MV, Grignon DJ, Pontes JE, Dhabuwala CB. High flow malignant priapism with isolated metastasis to the corpora cavernosa. Urology. 1998;51(2):324-6.

6. Lin YH, Kim JJ, Stein NB, Khera M. Malignant priapism secondary to metastatic prostate cancer: a case report and review of literature. Rev Urol. 2011;13(2):90-4.

7. Haddad FS. Penile metastases secondary to bladder cancer. Review of the literature. Urol Int. 1984;39(3):125-42.

8. Robey EL, Schellhammer PF. Four cases of metastases to the penis and a review of the literature. J Urol.1984;132(5):992-4.

9. Cherian J, Rajan S, Thwaini A, Elmasry Y, Shah T, Puri R. Secondary penile tumours revisited. Int Semin Surg Oncol. 2006;3:33.

10. Lau TN, Wakeley CJ, Goddard P. Magnetic resonance imaging of penile metastases: a report on five cases. Australas Radiol. 1999;43(3):378-81. 